\title{
Antidepressive response of inpatients with major depression to adjuvant occupational therapy: a case-control study
}

\author{
Marc-Andreas Edel ${ }^{1 *}$, Brian Blackwell ${ }^{1}$, Markus Schaub², Barbara Emons², Tanja Fox ${ }^{1}$, Friederike Tornau², \\ Bernward Vieten ${ }^{3}$, Patrik Roser ${ }^{1}$, Ida Sibylle Haussleiter ${ }^{1,2}$ and Georg Juckel ${ }^{1,2}$
}

\begin{abstract}
Background: Despite marked costs and limited evidence regarding effectiveness, occupational therapy (OT) is widely applied in psychiatric settings and financed by health insurance companies in European countries. This pilot study investigated the antidepressive effects of adjuvant OT for patients with major depression in a 6-week inpatient setting, stratified for females and males.
\end{abstract}

Methods: A total of 114 inpatients with major depression were assigned to either a standard OT group (using basic handcraft) or an active control group that played board games ( $2 \mathrm{~h}$ daily, 5 days a week). HAMD-21 scores were assessed as the primary outcome parameter after 3-6 weeks.

Results: The OT intervention was not superior to "board game" (BG) activities in reducing depressive symptoms. However, significant interaction effects were found in favor of the OT group regarding anxiety measures and other variables. Male participants displayed more significant interaction effects than female participants.

Conclusions: OT as an adjuvant short-term treatment for inpatients with major depression may be more efficacious than game interventions in terms of reducing anxiety and other symptoms, particularly in males.

Trial registration The study was registered in the EU Clinical Trials Register as a multicenter trial (EudraCT Number 2009016463-10; https://www.clinicaltrialsregister.eu/ctr-search/trial/2009-016463-10/DE\#A)

However, because of the elaborate setting requirements, the original study design with four centers was transformed into a solution with those two centers facilitating the pertinent resources. Furthermore, "mono-therapy with mirtazapine" was changed into "preferably mono-therapy with any antidepressant drug".

Keywords: Adjuvant occupational therapy, Major depression, Antidepressive effects

\section{Background}

Major depression is one of the most common and debilitating mental disorders. It causes enormous individual, social, and economic burden [18]. According to the German Information System of the Federal Health Monitoring, the diagnosis of a major depressive episode is the number one cause for inpatient treatment in German

\footnotetext{
*Correspondence: marc-andreas.edel@rub.de

${ }^{1}$ Department of Psychiatry, Psychotherapy and Preventive Medicine,

LWL University Hospital, Ruhr University Bochum, Alexandrinenstr. 1-3,

44791 Bochum, Germany

Full list of author information is available at the end of the article
}

psychiatric hospitals based on consistent data collected in the years 2000-2010 [5].

In several European countries, occupational therapy (OT) is known as Ergotherapie (from the Greek ergon $=$ work, exercise). In German-speaking countries, it is a traditional treatment that is most frequently applied in combination with other treatments, such as pharmacotherapy [14].

Occupational therapy is based on the positive relation between meaningful occupation and health, and views people as occupational beings [1]. "Occupational therapists should continue to be mindful of the humanistic 
ideals on which the profession was founded: the belief in the therapeutic value of meaningful occupation, the importance of the environment and of satisfactory interpersonal relationships, and balance in the daily routines of work, self-care and leisure" [13].

In Germany in particular, OT in inpatient psychiatric settings is mainly performed in group settings and is composed of four therapeutic facets. The first facet is the classical Ergotherapie that is the performance of various handcraft techniques with wood, stone, paper, and other materials. The second facet addresses the expression of inner states through drawing, painting, or modeling. The third facet focuses on the interactions and social skills of the group members while they are involved in common projects. The fourth facet concentrates on work performance and workplace reintegration aspects.

The extensive provision of OT for psychiatric patients in German-speaking countries causes marked costs, particularly in inpatient settings. According to Reuster [14], OT for inpatients in the Department of Psychiatry and Psychotherapy of the University of Dresden in 1998 was more expensive than pharmacotherapy for those patients. However, empirical data regarding the effectiveness and efficacy of OT in patients with mental disorders are lacking, and there are only few randomized controlled trials (RCTs) in this field.

Comparatively strong evidence exists for OT in community samples of people with dementia. Voigt-Radloff et al. [17] stated that the results of 5 of 7 RCTs suggested positive effects on activities of daily living (ADLs) or quality of life in persons with dementia or on their caregivers' skills, burden, and quality of life.

To date, OT for patients with schizophrenia has been investigated only in long-term RCTs $[3,4,6]$.

Schene et al. [15] were the first to perform a longterm RCT on OT (vs. treatment as usual, TAU) in outpatients with major depressive disorder; however, in that study, the OT intervention was not superior to TAU with respect to depression outcome. In a subsequent similar RCT (TAU + OT vs. TAU) in sick-listed employees with major depression, the workgroup focused on work participation as the primary outcome parameter, but significant benefits of adjuvant OT pertaining to a quick return to work, improvement of work-related coping and self-efficacy were not demonstrated. However, the OT group showed greater improvement in depression symptoms and an increased probability of long-term symptom remission and long-term return to work in good health [9].

Reuster [14] was the first to conduct a short-term RCT in 216 inpatients with major depression $(n=114$; $\left.\mathrm{n}_{+\mathrm{OT}}=63, n_{-\mathrm{OT}}=51\right)$, mania $\left(n=26 ; n_{+\mathrm{OT}}=16\right.$, $\left.n_{-\mathrm{OT}}=10\right)$, and schizophrenia $\left(n=76 ; n_{+\mathrm{OT}}=41\right.$,
$\left.n_{-\mathrm{OT}}=35\right)$. That author investigated the effects of daily add-on standard OT (performance and training of handcraft techniques using wood, stone, paper, and other materials) versus self-instructed unspecific activities on psychopathological variables over four weeks within a multimodal clinical setting. A significant reduction of symptomatology was only observed in the patients with major depression (43.9\% decrease in the Bech Rafaelsen Melancholia Scale score vs. a $27.5 \%$ decrease in the activity group after 4 weeks). However, that study had multiple methodological problems; it lacked a primary outcome parameter, an assessment of functioning in $\mathrm{ADL}$, and control for confounders, such as drug therapy, psychoeducative and psychotherapeutic sessions, and other covariates, such as exercise therapy.

Because the above-mentioned study by Reuster is the only investigation of short-term OT in inpatients with major depression to date, we felt inspired to study this topic further.

We planned and performed a pilot RCT in inpatients with moderate-to-severe major depression that compared a standard OT group program, i.e., everyday performance of handcraft activities, to a board game (BG) group as a semi-active control. In both groups, the interventions were in addition to basic antidepressant drug treatment and short daily supportive talks with staff members.

We avoided the major limitations of Reuster's study by defining the change in the total score of the Hamilton Depression Rating Scale (HAMD) from before to after the interventions as the primary outcome parameter. Moreover, we applied secondary assessments of psychopathological symptoms, such as anxiety, and we included a specific OT assessment (Ergo-Assess ${ }^{\mathrm{TM}}$ ) of functioning in ADLs, and we controlled for confounders (antidepressant drugs and psychiatric comorbidity).

We expected that the adjuvant OT intervention would result in significantly greater effects indicating decrease in depressive symptoms and secondary psychopathological characteristics. Furthermore, we hypothesized superiority of the OT over the BG intervention with respect to improvements in ADL and social functioning. Additionally, gender differences regarding effects indicating possible improvements were explored.

\section{Methods \\ Participants}

A total of 131 inpatients who experiences a moderate or severe major depressive episode diagnosed according to the DSM-IV criteria (moderate, 296.22, or severe episode without psychotic features, 296.23; recurrent major depressive disorder: moderate, 296.32, or severe episode without psychotic features, 296.33) were recruited from three similar inpatient units of two German psychiatric 
clinics and assessed for eligibility for participation in this study. All diagnoses were established using the Structured Clinical Interview for the DSM-IV (SCID I/II). Of the 131 patients who were screened, 14 did not meet the inclusion criteria and three refused to participate. Finally, 114 patients (55\% female, mean age $45.7 \pm 11.8$ years) were randomly (by the block random method) assigned to either the experimental (OT) or the active comparison group (board game group, BG). During the first 3 weeks, 11 (19.3\%) OT participants and 21 (36.8\%) BG participants dropped out for motivational reasons. $46 \mathrm{OT}$ participants and 36 BG participants participated in the study for at least three weeks $(n=82)$ and 29 OT participants and 22 BG participants completed the study ( 6 weeks, $n=51$ ). The data were processed in per-protocol analyses.

No significant group differences emerged regarding sex, age, education, marital status, intelligence, axis-I or axis-II comorbidity, number of psychoactive drugs, or number of antidepressants. However, as the only particular difference between study groups, the female participants in the OT group took significantly more psychoactive drugs $(p=0.020)$ than the female participants in the BG group (Table 1). No overall gender differences were found between study groups concerning intelligence, axis-I or axis-II comorbidity, number of psychoactive drugs, or number of antidepressants.

Over $80 \%$ of the participants took at least one antidepressant drug before admission. Of these participants, only about $20 \%$ had only one antidepressant.

All participants gave written informed consent, and the Ethics Committee of the Medical Faculty of the Ruhr University Bochum approved the study (No. 3626-10FF).

\section{Study design, interventions, and inclusion/exclusion criteria}

The study was primarily designed as a 6-week pilot RCT with a block randomization. However, block size could not be fixed randomly in this study, but the trialists allocated blocks of three, four, or five participants to the groups alternately, according to the availability of eligible patients.

Table 1 Group comparisons (demographic and clinical data)

\begin{tabular}{|c|c|c|c|c|c|}
\hline & Occupational therapy & Board game group & Chi square ${ }^{a} / t^{b}$ & df & $p$ \\
\hline \multicolumn{6}{|l|}{ Demographic data } \\
\hline$N=82$ & $n=46$ & $n=36$ & & & \\
\hline $\operatorname{Sex}(f: m)$ & $26: 20$ & 18:18 & $0.38^{\mathrm{a}}$ & 1 & 0.539 \\
\hline Age, years (SD) & $46.8(11.8)$ & $44.8(11.7)$ & $0.84^{b}$ & 81.8 & 0.401 \\
\hline Age, females (SD) & $45.7(10.7)$ & $45.4(13.3)$ & $0.11^{\mathrm{b}}$ & 40.4 & 0.910 \\
\hline Age, males (SD) & $48.2(13.3)$ & $44.1(9.8)$ & $1.18^{\mathrm{b}}$ & 31.5 & 0.247 \\
\hline Age groups & 6 groups ranging from 18 to 70 years of age & & $2.74^{\mathrm{a}}$ & 5 & 0.740 \\
\hline Educational level & $\begin{array}{l}11 \text { categories ranging from 'no degree' } \\
\text { to 'university degree' }\end{array}$ & & $17.3^{\mathrm{a}}$ & 10 & 0.068 \\
\hline Marital status & 4 categories (single, married, divorced, widowed) & & $577^{\mathrm{a}}$ & 3 & 0.124 \\
\hline \multicolumn{6}{|l|}{ Clinical data } \\
\hline Intelligence, MWT-B, raw data (SD) & $29.1(14.8)$ & $28.1(4.8)$ & $0.50^{\mathrm{b}}$ & 71.2 & 0.617 \\
\hline Intelligence, females (SD) & $30.3(9.0)$ & $27.7(4.7)$ & $0.77^{\mathrm{b}}$ & 37.7 & 0.448 \\
\hline Intelligence, males (SD) & $27.5(4.8)$ & $28.5(5.0)$ & $-0.70^{\mathrm{b}}$ & 37 & 0.487 \\
\hline Axis-I disorders (SD) & $1.7(0.8)$ & $1.5(0.7)$ & $0.18^{b}$ & 75 & 0.861 \\
\hline Axis-I disorders, females (SD) & $1.8(0.9)$ & $1.4(0.6)$ & $1.62^{\mathrm{b}}$ & 35.8 & 0.112 \\
\hline Axis-I disorders, males (SD) & $1.3(0.6)$ & $1.7(0.8)$ & $-1.74^{b}$ & 36 & 0.090 \\
\hline Axis-II disorders (SD) & $0.2(0.4)$ & $0.1(0.3)$ & $0.60^{b}$ & 75 & 0.550 \\
\hline Axis-II disorders, females (SD) & $0.2(0.4)$ & $0.1(0.3)$ & $0.22^{b}$ & 39.7 & 0.827 \\
\hline Axis-II disorders, males (SD) & $0.2(0.4)$ & $0.1(0.3)$ & $0.64^{b}$ & 36 & 0.524 \\
\hline Number of psychoactive drugs (SD) & $2.6(1.4)$ & $2.4(1.3)$ & $0.63^{b}$ & 72 & 0.529 \\
\hline Number of psychoactive drugs, females (SD) & $2.6(1.3)$ & $2.0(0.8)$ & $2.40^{\mathrm{b}}$ & 42 & 0.020 \\
\hline Number of psychoactive drugs, males (SD) & $2.4(1.4)$ & $2.9(1.8)$ & $-0.99^{\mathrm{b}}$ & 36 & 0.331 \\
\hline Number of antidepressants (SD) & $1.8(1.0)$ & $1.7(0.8)$ & $0.20^{b}$ & 77.8 & 0.848 \\
\hline Number of antidepressants, females (SD) & $1.8(0.8)$ & $1.6(0.8)$ & $1.02^{\mathrm{b}}$ & 38.7 & 0.311 \\
\hline Number of antidepressants, males (SD) & $1.7(1.2)$ & $1.9(0.72)$ & $-0.64^{b}$ & 36 & 0.524 \\
\hline
\end{tabular}

a relates to Pearson's Chi-square tests

b relates to $t$ tests 
Basic handcraft is the core OT activity in Germanspeaking countries. Therefore, the primary or experimental intervention comprised standardized performance of basic handcraft activities, such as painting and crafting with wood, stone, and other materials.

Board game activities were used for control condition. The resemblance of such activities with OT in a stricter sense should improve the acceptability of the control intervention, since many patients claim OT as an essential part of inpatient treatments.

Both interventions were conducted $2 \mathrm{~h}$ daily, 5 days a week. Only one handcraft activity (either crafting or painting) or board game (like Monopoly or cards, involving more than two persons, thus no chess or Scrabble) was performed in each 2-h session. Both interventions were provided for groups with 6-8 patients and conducted by professional occupational therapists. No cognitive or talk therapy was added to the interventions, but a basic antidepressant drug treatment and supportive or psychoeducative talks up to $20 \mathrm{~min}$ per day with staff members were allowed.

The inclusion criteria were the following: 18- to 65-year-old inpatients with moderate or severe major depression without psychotic or catatonic features (moderate, 296.22, or severe episode without psychotic features, 296.23; recurrent major depressive disorder: moderate, 296.32, or severe episode without psychotic features, 296.33) and a Hamilton Depression Rating Scale (HAMD-21) score $\geq 18$. Any antidepressant medication was allowed, if possible as a mono-therapy. Z-drugs were permitted to treat sleep problems. In case of restlessness or agitation, promethazine (up to $75 \mathrm{mg}$ per day), lorazepam (up to $3 \times 1 \mathrm{mg}$ per day) or quetiapine (up to $100 \mathrm{mg}$ per day) could be prescribed.

The exclusion criteria were the following: contraindications for antidepressants; currently at risk of suicide; and a DSM-IV diagnosis of any of the following: dementia, schizophrenia spectrum disorders, cluster A and cluster B personality disorders, substance use disorders (abuse and dependence), eating disorders (anorexia and bulimia nervosa); acute, serious, or unstable medical conditions; and pregnancy in females.

\section{Outcome measures}

The primary outcome parameter was decrease in depressivity as measured by the Hamilton Depression Rating Scale, HAMD-21 [7]. The Beck Depression Inventory, BDI [2], was used as a secondary outcome measure. Compared to the (interviewer-rating) HAMD, the (selfrating) $\mathrm{BDI}$ assesses rather subjective depressivity, and reductions of BDI scores during therapy may depend on personality traits, particularly introversion and neuroticism, to a larger extent, than do changes in HAMD scores. Therefore, complementary performance of both instruments may be useful [16]. State anxiety was measured using the Hamilton Anxiety Rating Scale, HAMA [8]. Furthermore, the Personal and Social Performance Scale, PSP, an interviewer-rating instrument, was used to assess four features of social functioning (socially useful activities; personal and social relationships; self-care; and disturbing and aggressive behavior) over a one-month period $[12,11]$. These scales were applied for screening and baseline ratings and for the follow-up assessments at 3-9 weeks after baseline. A physician and a psychologist from our work group carried out the assessments. The software package Ergo-Assess ${ }^{\mathrm{TM}}$ [10] was used to assess functioning in activities of daily living (ADL) in the six domains of the International Classification of Functioning, Disability and Health, ICF [19]: (1) activities of physical self-care, (2) activities of independent living, (3) neuropsychological functioning, (4) psychosocial functioning, (5) sensomotoric functions, and (6) basic work activities. In contrast to the other instruments, ErgoAssess was used at 1-6 weeks, as opposed to 3-6 weeks, after baseline by a professional occupational therapist.

\section{Data analysis}

Statistical analyses were carried out using the Statistical package for the Social Sciences $\left(\right.$ SPSS $^{\mathrm{TM}}$ ), version 20 for Mac, IBM Corp., Armonk, New York, United States. The one-sample Kolmogorov-Smirnov test was used to confirm that all interval-scaled variables were normally distributed. Group comparisons in respect to gender, age, education, depressive symptomatology, comorbidity, and medication were conducted using $t$ tests and Pearson's Chi-square tests. A reduction of the HAMD-21 total score of $\geq 50 \%$ from baseline was defined as 'antidepressive response,' and a HAMD-21 total score of $\leq 7$ was defined as remission. The groups were compared with respect to these HAMD factors by performing Pearson's Chi-square tests. Possible treatment effects were investigated using general linear models (GLM) with repeated measures analyses of variance. Age, IQ, axis-I and axis-II comorbidity, number of psychoactive drugs, and number of antidepressants were taken into account as covariates. Cohen's measure of sample effect size for comparing two samples means, i.e., pre- and post-means, was then used to assess possible treatment effects. Results with $p<0.05$ were considered statistically significant.

\section{Results}

\section{Antidepressive response and remission}

No significant group differences were found in terms of antidepressive response or remission after three- and six-week treatment. Antidepressive response was found in 10 participants $(21.7 \%)$ of the OT group $(n=46)$ 
and 13 participants $(36.1 \%)$ of the BG group $(n=36)$ after 3 weeks, and in 19 participants $(65.5 \%)$ of the OT group $(n=29)$ and 12 participants $(54.5 \%)$ of the BG group $(n=22)$ after 6 weeks. Remission was found in nine participants $(19.6 \%)$ in the OT group vs. five participants $(13.9 \%)$ in the BG group (Chi-square $=0.536$, $d f=1, p=0.464)$ after 3 weeks, and in eight participants $(27.6 \%)$ in the OT group vs. nine participants $(40.9 \%)$ in the BG group after 6 weeks (Chi-square $=0.777, d f=1$, $p=0.378$ ).

\section{Primary outcome parameter}

The GLM analysis did not find any significant time-bygroup interaction effects regarding the primary outcome parameter HAMD total score (after 3 weeks: $F=0.141$, $p=0.709$; after 6 weeks: $F=0.177, p=0.828$ ). This indicates that neither group reached antidepressive superiority.

\section{Secondary outcome parameters}

A significant time-by-group interaction effect regarding the HAMA total score in males after three weeks was observed which suggests superiority of the OT intervention over the BG intervention $(F=5.226, p=0.031$; $d=1.23$ vs. 0.48 ) (Table 2). At 6 weeks, no significant interaction effect was found. No other significant interaction effects were observed regarding the other total scores (BDI, PSP and ErgoAssess).

\section{Subscale parameters \\ Comparison after 3 weeks}

The following significant time-by-group interactions were observed: loss of interest (BDI 12) in favor of the OT group $(F=13.494, p=0.001 ; d=0.95$ vs. 0.00$)$ in the male participants; disturbed sleep pattern (BDI 16) in favor of the BG group $(F=4.983, p=0.029 ; d=0.92$ vs. 0.31) in both genders; loss of sexual interest (BDI 22) in favor of the OT group in the male participants $(F=5.017, p=0.034 ; d=0.22$ vs. 0.00$)$; depressed mood (HAMA 6) in favor of the OT group in both genders $(F=4.190, p=0.044 ; d=1.20$ vs. 0.79$)$; and self-care (PSP C) in favor of the BG group in the female participants $(F=5.213, p=0.029 ; d=0.44$ vs. 0.00$)$ (Table 2$)$.

\section{Comparison after 6 weeks}

The following significant time-by-group interaction effects were found subscales of the various inventories assessed: depersonalization and derealization (HAMD $19)$ in favor of the BG group in all participants $(F=4.321$, $p=0.044 ; d=0.71$ vs. 0.00$)$ and in the subgroup of male participants $(F=4.944, p=0.039 ; d=0.83$ vs. 0.00$)$; loss of energy (BDI 15) in favor of the OT group in all participants $(F=5.095, p=0.030 ; d=1.05$ vs. 0.46$)$; disturbed sleep pattern (BDI 16) in favor of the OT group in the subgroup of female participants $(F=6.415, p=0.025$; $d=0.92$ vs. 0.51); loss of sexual interest (BDI 22) in favor of the OT group in all participants $(F=11.908$, $p=0.001 ; d=0.36$ vs. 0.00 ) and in the subgroup of male participants $(F=6.642, p=0.028$; $d=0.57$ vs. 0.00$)$; anxious behavior during the interview (HAMA 14) in favor of the OT group in the subgroup of male participants $(F=6.301, p=0.022 ; d=1.26$ vs. 0.41$)$; and basic work skills (ErgoAssess 3 ) in favor of the OT group in all participants $(F=6.344, p=0.017 ; d=1.83$ vs. 0.16$)$ (Table 3).

\section{Discussion}

Data on adjuvant occupational therapy (OT) in inpatient psychiatric settings is greatly lacking. In fact, there have only been two studies on this topic $[6,14]$ to date. Only one trial by Reuster evaluated the effects of short-term adjuvant OT; that study was on inpatients with schizophrenia, mania, and major depression. Unfortunately, that study has not been published in PubMed, and it is the only available work that is comparable to ours.

In this study, our purpose was to investigate the effects of short-term adjuvant OT in patients with a mental disorder of considerable epidemiologic and clinical relevance, i.e., major depression, in an inpatient (i.e., costly) setting. In German-speaking countries, OT is broadly applied and generally financed by health insurance companies. The main difficulty in designing this study was that a simple comparison of pharmacotherapy alone (preferably with a single drug) to pharmacotherapy plus OT was not possible because the standard psychiatric inpatient settings in German-speaking countries provide pharmacotherapy, psycho-education and psychotherapy in single and group settings and exercise therapy, different occupational therapies, and other treatments. Therefore, we attempted to merge the demands of patients and the requirements of health insurance companies into a study design that was as simple as possible and, most importantly, had the least possible number of confounders.

Our main finding was that the interventional OT group was not superior to the control board game (BG) group with respect to our primary outcome measures: The study did not show any reduction of depressivity and percentage of remissions as measured by the Hamilton Depression Scale (HAMD-21). However, some significant time-by-group effects indicated a superiority of the OT intervention over the BG intervention with respect to anxiety, i.e., reductions in anxiety in general (HAMA total score) in male participants after three weeks, depressed mood (HAMA subscale) in participants of both genders after three weeks, and anxious behavior 


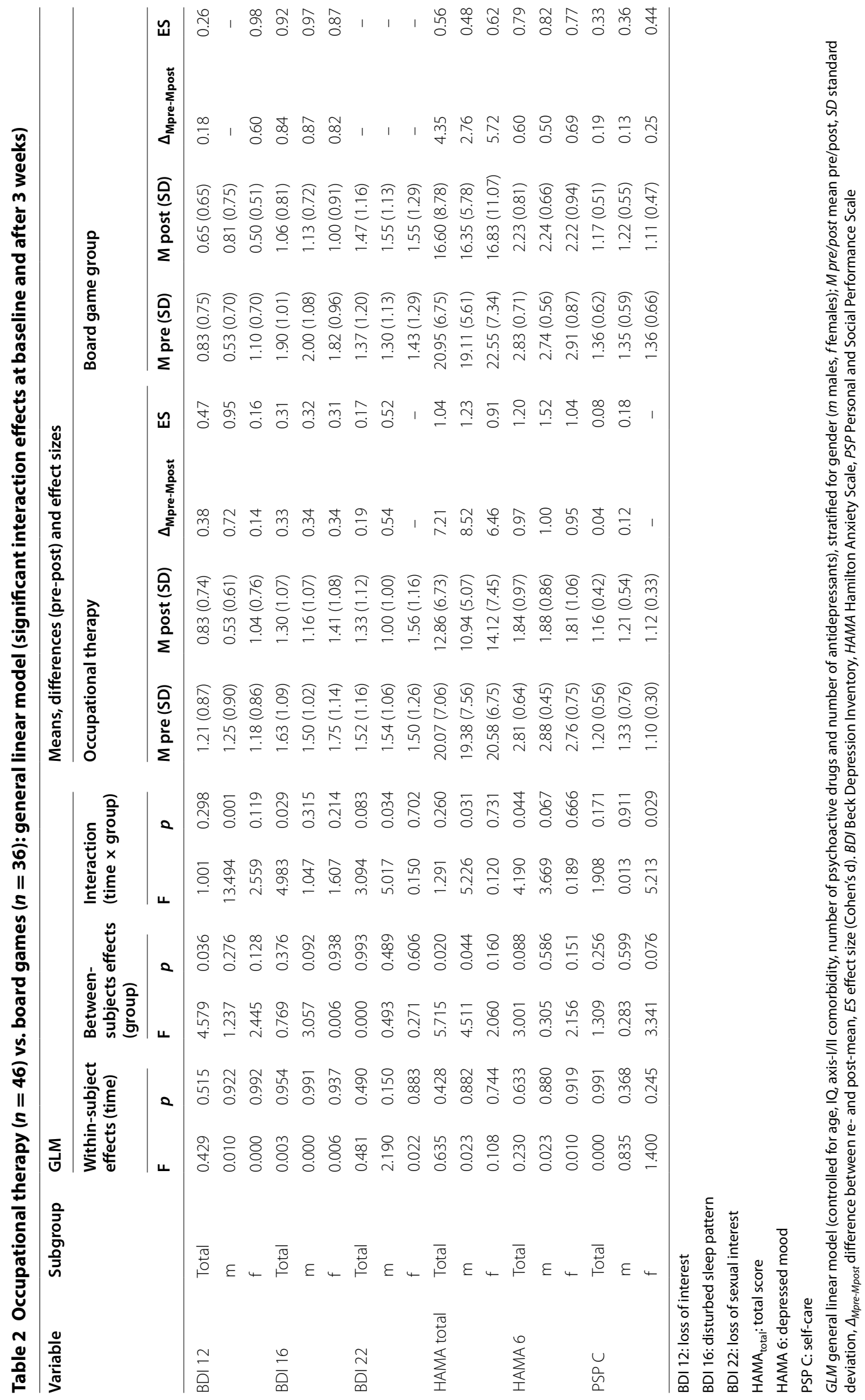




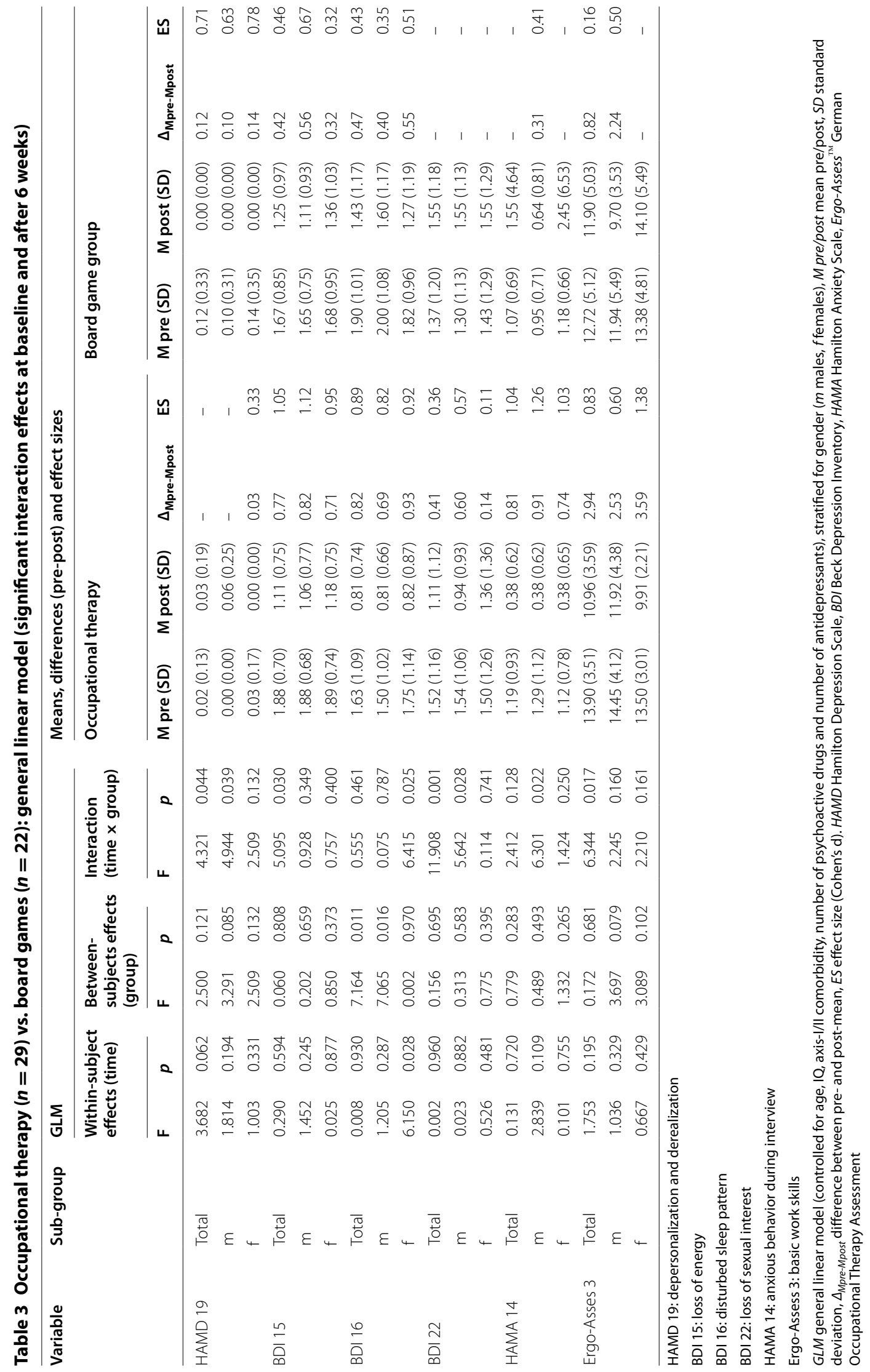


during the interview (HAMA subscale) in male participants after six weeks.

Moreover, significant interaction effects in regards to some subscales of the Beck Depression Inventory (BDI) indicated the superiority of OT over BG, including loss of general interest and loss of sexual interest in males after 3 weeks, loss of energy in all participants after six weeks, disturbed sleep pattern in females after 6 weeks, and loss of sexual interest in both genders after 6 weeks. However, a significant interaction effect with respect to disturbed sleep pattern in participants of both genders after 3 weeks suggested a superiority of BG over OT. Other measures in favor of BT included self-care (from the Personal and Social Performance Scale) after 3 weeks in the female participants and depersonalization and derealization (from the HAMD) after 6 weeks in participants of both genders.

Finally, basic work skills (assessed with Ergo-Assess ${ }^{\mathrm{TM}}$ ) improved significantly more in the OT participants of both genders after 6 weeks. Effect sizes with respect to the superior group, i.e., predominantly the OT group, were mainly in the high range $(d>0.8)$.

There were several limitations to this study: We faced difficulties concerning the comparability of the two groups; for example, patients found the OT intervention much more pleasant and effective than the BG activities, which was reflected by a far greater dropout rate during the first 3 weeks in the BG group, $36.8 \%$, compared to the OT group, $19.3 \%$.

The ("semi-active") BG group was not a true control group, such as a placebo or waitlist group; thus, we can only discuss 'effects' but not 'efficacy' of the OT intervention in comparison to the BG activities.

For comparability reasons, each group intervention was completely structured and standardized as to content and procedure, which entailed limited performance of individually meaningful activities and accomplishment of personal goals. Thus, the OT intervention was limitedly representative of standard OT group interventions for inpatients. Moreover, our "OT" intervention is by no means representative of occupational therapy in general.

We were not able to explain why BDI but not HAMD scores decreased during the intervention. Changes in BDI scores rather than HAMD scores emerged to be associated with personality features like introversion and neuroticism [16]; however, this study lacked assessment of personality traits.

Insufficient qualitative and process-related assessment of pharmacotherapy represents a major shortcoming of this study. However, the numbers of psychoactive and antidepressant drugs were considered as covariates, and $80 \%$ of the participants were known to enter the study taking at least one antidepressant.
Finally, the study was underpowered, as it had an even smaller study sample size than that of Reuster's investigation (82 participants in our study vs. 114 participants in Reuster's study). Thus, this study has to be regarded as a pilot project.

\section{Conclusions}

In conclusion, the OT group in our study showed more significant effects indicating improvement with respect to features of anxiety, loss of energy, sexual and general interest, and work skills than the BG group. Moreover, the results of this study suggest a greater benefit of the OT intervention in males than in females.

Together, our results suggest that adjuvant standard occupational treatment may be superior to mere board game activities and may be a feasible adjunct therapy to pharmacotherapy (and possibly other treatments) in a psychiatric short-term setting for inpatients with major depression.

\section{Abbreviations \\ ADL: activities of daily living; BDI: Beck Depression Inventory; BG (group): board game (group); DSM-IV: Fourth edition of the Diagnostic and Statistical Manual of the American Psychiatric Association; Ergo-Assess ${ }^{\mathrm{TM}}$ : instrument for the assessment of occupational therapy outcome; GLM: general linear model; HAMA: Hamilton Anxiety Rating Scale; HAMD (-21): Hamilton Depression Rating Scale (with 21 items); ICF: International Classification of Functioning, Disability and Health; OT (group): occupational therapy (group); PSP: scale personal and social performance scale; RCT: randomized controlled trial; SCID: Structured Clinical Interview for the DSM-IV; SPSS: statistical package for the social sciences; TAU: treatment as usual; WHO: World Health Organization.}

\section{Authors' contributions}

All of us authors made substantial contributions to the conception and design or analysis and interpretation of data. Furthermore, all of us were involved substantially in drafting or revising the article for important and novel content, and gave approval of the final version to be submitted. Particularly, MAE, BV, PR, ISH and GJ were substantially involved in the planning of the study design, providing treatment facilities, supervising occupational therapists, evaluating study results, and writing of the paper. $\mathrm{BB}, \mathrm{MS}, \mathrm{BE}$, and FT coordinated the allocation of participants and took care of the assessment, evaluation, analysis, and presentation of data; moreover, the colleagues were involved in drafting and revising the manuscript. TF was part of our study team. As the leading occupational therapist, she was involved in the conceptualization of the study, moreover in active performance and supervising colleagues concerning occupational therapy and "game therapy" in the study groups, and in the final presentation of results. All authors have read and approved the final manuscript.

\section{Author details}

${ }^{1}$ Department of Psychiatry, Psychotherapy and Preventive Medicine, LWL University Hospital, Ruhr University Bochum, Alexandrinenstr. 1-3, 44791 Bochum, Germany. ${ }^{2}$ Institute of Mental Health, LWL University Hospital Bochum, Bochum, Germany. ${ }^{3}$ LWL Hospital Paderborn, Paderborn, Germany.

\section{Acknowledgements}

We thank Birgit Zander, Petra Nengelken, and Bettina Finger for acquisition of the literature we have used and referred to in our paper, and for drafting the manuscript. Moreover, many thanks to the colleagues of our inpatient occupational therapy unit for therapeutic activities and support, and to all patients who participated in the study.

\section{Competing interests}

The authors declare that they have no competing interests. 


\section{Availability of data and materials}

All data generated or analyzed during the current study available from the corresponding author on reasonable request.

\section{Ethics approval and consent to participate}

All participants gave written informed consent, and the Ethics Committee of the Medical Faculty of the Ruhr University Bochum approved the study (No. 3626-10FF). Our work has been performed in accordance with the Declaration of Helsinki.

\section{Funding}

The study was financed by the participating hospitals without any external sponsorship.

Received: 12 October 2016 Accepted: 22 December 2016

Published online: 03 January 2017

\section{References}

1. American Occupational Therapy Association. Occupational therapy practice framework: domain and process. Am J Occup Ther. 2008:62:625-83.

2. Beck AT, Ward CH, Mendelson M, Mock J, Erbaugh J. An inventory for measuring depression. Arch Gen Psychiatry. 1961;4:561-71.

3. Buchain PC, Vizzotto AD, Henna Neto J, Elkis H. Randomized controlled trial of occupational therapy in patients with treatment-resistant schizophrenia. Rev Bras Psiquiatr. 2003;25:26-30.

4. Cook S, Chambers E, Coleman JH. Occupational therapy for people with psychotic conditions in community settings: a pilot randomized controlled trial. Clin Rehabil. 2009. doi:10.1177/0269215508098898.

5. Die Gesundheitsberichterstattung des Bundes [Transl.: The German Information System of the Federal Health Monitoring]. Wiesbaden: Statistisches Bundesamt; 2006. http://www.gbe-bund.de. Accessed 5 Aug 2016

6. Foruzandeh N, Parvin N. Occupational therapy for inpatients with chronic schizophrenia: a pilot randomized controlled trial. Jpn J Nurs Sci. 2013 doi:10.1111/j.1742-7924.2012.00211.x.

7. Hamilton M. A rating scale for depression. J Neurol Neurosurg Psychiatry. 1960;23:56-62.
8. Hamilton M. The assessment of anxiety states by rating. $\mathrm{Br} J$ Med Psychol. 1959:32:50-5.

9. Hees HL, de Vries G, Koeter MW, Schene AH. Adjuvant occupational therapy improves long-term depression recovery and return-to-work in good health in sick-listed employees with major depression: results of a randomised controlled trial. Occup Environ Med. 2013;70:252-60.

10. Heiss HW, Voigt-Radloff S, Schochat T. Occupational Therapy Assessment (OTA): validity and reliability for adults of various ages. Eur J Ger. 2003:5:23-9.

11. Juckel G, Schaub D, Fuchs N, Naumann U, Uhl I, Witthaus H, Hargarter L, Bierhoff HW, Brüne M. Validation of the Personal and Social Performance (PSP) Scale in a German sample of acutely ill patients with schizophrenia. Schizophr Res. 2008. doi:10.1016/j.schres.2008.04.037.

12. Morosini PL, Magliano L, Brambilla L, Ugolini S, Pioli R. Development, reliability and acceptability of a new version of the DSM-IV Social and Occupational Functioning Assessment Scale (SOFAS) to assess routine social functioning. Acta Psychiatr Scand. 2000;101:323-9.

13. Paterson CF. History of occupational therapy in mental health. In: Bryant W, Fieldhouse J, Bannigan K, editors. Creek's Occupational Therapy and Mental Health. 5th ed. London: Churchill/Livingstone (Elsevier); 2014

14. Reuster T. Effektivität der Ergotherapie im psychiatrischen Krankenhaus [Transl.: Effectiveness of occupational therapy in the psychiatric hospital]. Vol. 112. Darmstadt; 2006

15. Schene AH, Koeter MW, Kikkert MJ, Swinkels JA, McCrone P. Adjuvant occupational therapy for work-related major depression works: randomized trial including economic evaluation. Psychol Med. 2007:37:351-62.

16. Schneibel R, Brakemeier EL, Wilbertz G, Dykierek P, Zobel I, Schramm E. Sensitivity to detect change and the correlation of clinical factors with the Hamilton Depression Rating Scale and the Beck Depression Inventory in depressed inpatients. Psychiatry Res. 2012;198:62-7.

17. Voigt-Radloff S, Ruf G, Vogel A, van Nes F, Hüll M. Occupational therapy for elderly: evidence mapping of randomised controlled trials from 2004 to 2012. Z Gerontol Geriatr. 2015;48:52-72.

18. World Health Organization. Depression, a global public health concern. Geneva: World Health Organization; 2012.

19. World Health Organization. International Classification of Functioning Disability and Health. Geneva: World Health Organization; 2001.

\section{Submit your next manuscript to BioMed Central and we will help you at every step:}

- We accept pre-submission inquiries

- Our selector tool helps you to find the most relevant journal

- We provide round the clock customer support

- Convenient online submission

- Thorough peer review

- Inclusion in PubMed and all major indexing services

- Maximum visibility for your research

Submit your manuscript at www.biomedcentral.com/submit
BioMed Central 